\title{
Writing ROOT Data in Parallel with TBufferMerger
}

\author{
Guilherme Amadio ${ }^{1, *}$, Philippe $\mathrm{Canal}^{2}$, Enrico Guiraud ${ }^{1}$, and Danilo Piparo ${ }^{1}$ \\ ${ }^{1}$ CERN, Geneva, Switzerland \\ ${ }^{2}$ Fermi National Accelerator Laboratory, Batavia, IL, USA
}

\begin{abstract}
Experiments at the Large Hadron Collider (LHC) produce tens of petabytes of new data in ROOT format per year that need to be processed and analysed. In the next decade, following the planned upgrades of the LHC and its detectors, this data production rate is expected to increase at least ten-fold. Therefore, optimizing the ROOT I/O subsystem is of critical importance to the success of the LHC physics programme. This contribution presents ROOT's approach for writing data from multiple threads to a single output file in an efficient way. Technical aspects of the implementation-the TBufferMerger class - and programming model examples are described. Measurements of runtime performance and the overall improvement relative to the case of serial data writing are also discussed.
\end{abstract}

\section{Introduction}

Most of the tens of petabytes of new data produced each year at the Large Hadron Collider (LHC) is stored in ROOT's file format [1]. At the current rate, this translates to about 30 petabytes of ROOT data per year [2] that needs to be stored by physicists for later analysis. In the next decade, a sudden increase of this rate to hundreds of petabytes is expected at the beginning of Run 3. Since the amount of computational resources required to analyse data is proportional to the total volume of data to be analysed, it has become clear that under the flat or mildly increasing budget prospects for the coming years we will suffer from a shortage of computational resources if no measures are taken to optimize all HEP software. ROOT has a central role in HEP data analysis and as such has undergone efforts in several fronts to increase data processing performance. Some of these efforts have been discussed before in [3]. Here we shall focus on the latest developments on parallel data writing with ROOT using the TBufferMerger class.

\section{The TBufferMerger Class}

The TBufferMerger class, introduced in ROOT 6.10, allows users to write ROOT data in parallel to a single output file. Two use cases inspired the development of TBufferMerger: parallel writing of detector simulation and track reconstruction data, and parallel processing of existing datasets with ROOT's RDataFrame, for example, when applying filters, creating derived quantities, and then saving the results to a new file for further processing.

\footnotetext{
*e-mail: amadio@cern.ch
} 
Since users are already familiar with ROOT's interfaces for creating and filling ROOT datasets, the main concern when designing TBufferMerger was to not diverge too much from existing interfaces in order to minimize the amount of changes required to convert sequential code to write data in parallel using the new class. ROOT already has the TMemFile class that can be used to store files in memory, and the TFileMerger class to merge files on disk. In the initial implementation of TBufferMerger, we adapted TMemFile to write data into a queue that is then merged by TFileMerger in a background thread into the output file on disk. The diagram in Figure 1 shows how this works in practice. The red dot represents a sentinel indicating that the output file is complete and should be closed.

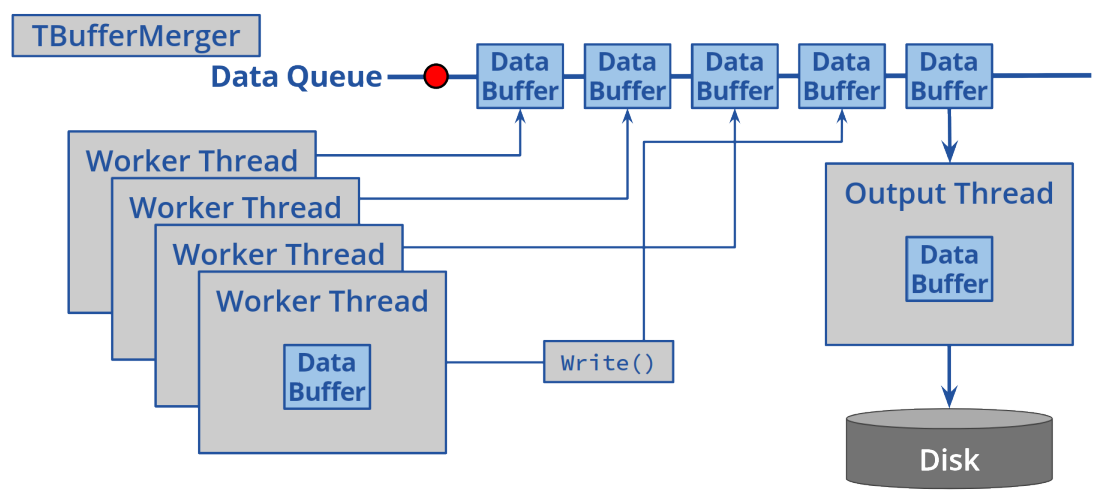

Figure 1: Initial implementation of TBufferMerger. Worker threads place data in the queue, while the output thread merges buffers one by one into the output file.

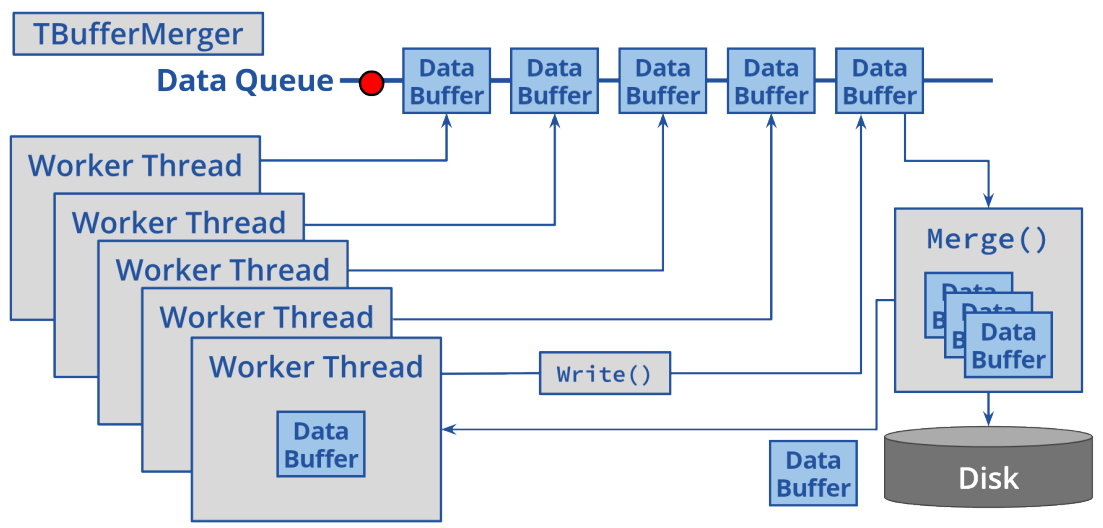

Figure 2: Current implementation of TBufferMerger. Worker threads/tasks place data in the queue. Upon writing its own data to the queue, each thread or task checks if a merge should be triggered and performs the merge if necessary, without spawning new threads or tasks in the background.

After being integrated into ROOT and tested by CMS for writing out reconstruction data in parallel [4], it became clear that despite the performance gains, the choice of using a background thread led to some inconveniences, like oversubscription of machines where reconstruction jobs were running within the CMSSW framework. Therefore, the TBufferMerger 


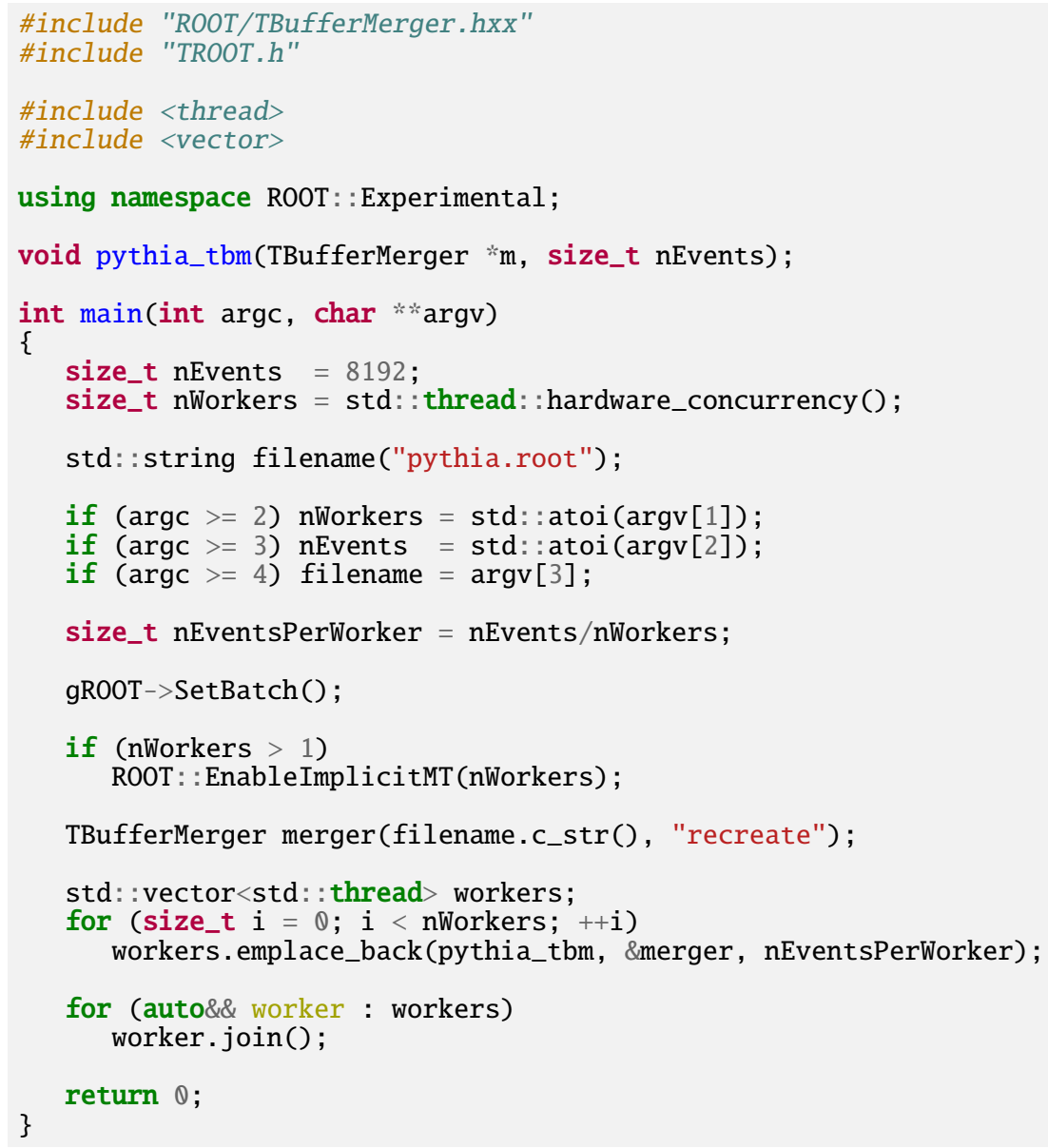

Listing 2: Example of how to create workers for TBufferMerger using $\mathrm{C}++11$ threads.
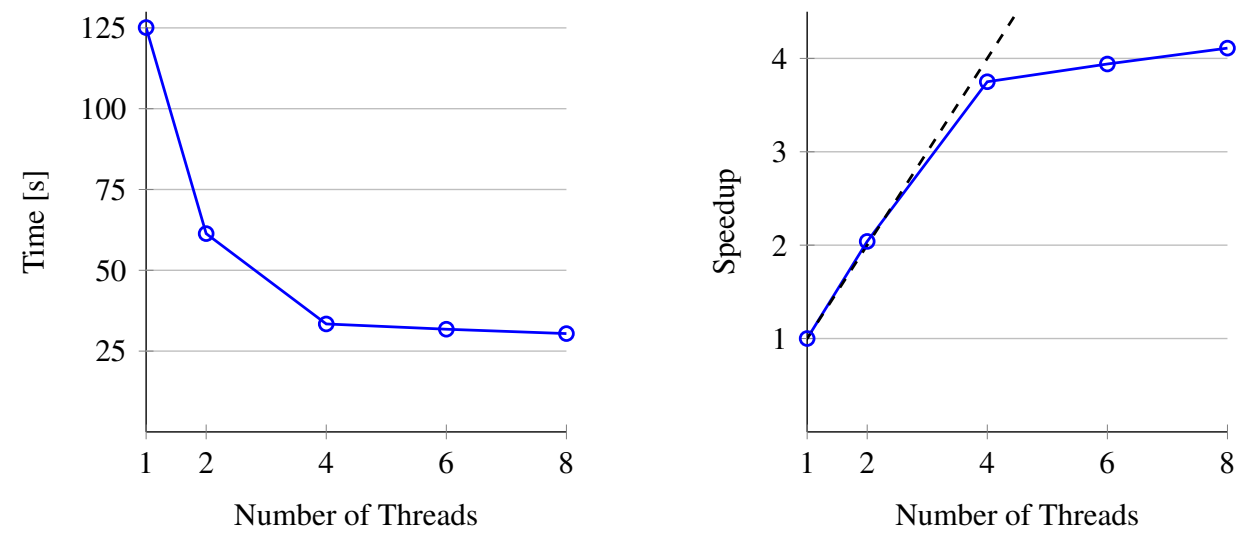

Figure 3: Performance of Pythia event generation example on a quad-core desktop machine (Intel Core i7 $6700 \mathrm{CPU}$ at $3.4 \mathrm{GHz}$ ). 


\section{Parallel Data Writing Benchmarks}

In this section we present performance benchmarks using TBufferMerger in various scenarios. All benchmarks have been run on a machine with an Intel@ Core ${ }^{\mathrm{TM}} \mathrm{i} 7-7820 \mathrm{X}$ processor ( 8 cores, 11M Cache, up to $4.30 \mathrm{GHz}$ ) and $32 \mathrm{~GB}$ of RAM, where a release build of ROOT has been compiled with GCC 8.1 using the native instruction set (Skylake AVX-512). The output disks used were a WD Black Hard Disk Drive (1TB), and a Samsung Evo 960 NVMe SSD (256GB). For comparison, we have also tested writing out to system memory (using an output file in tmpfs).

The first benchmark consists in creating a dataset of $\approx 1 \mathrm{~GB}$ and saving it in parallel using different compression algorithms and output media. The dataset contains a single column of data with randomly generated floating point numbers in the range [0, 1]. The LZ4 compression algorithm is the fastest but produces larger files than ZLIB and LZMA. The ZLIB algorithm has a good balance between compression ratio and compression speed, and the LZMA algorithm has the best compression ratio but has also the slowest compression speed. Results are shown in Figure 4. When using LZMA compression, the benchmark is CPU-bound, and shows nearly ideal scaling. In other configurations, performance is limited by the output bandwidth.
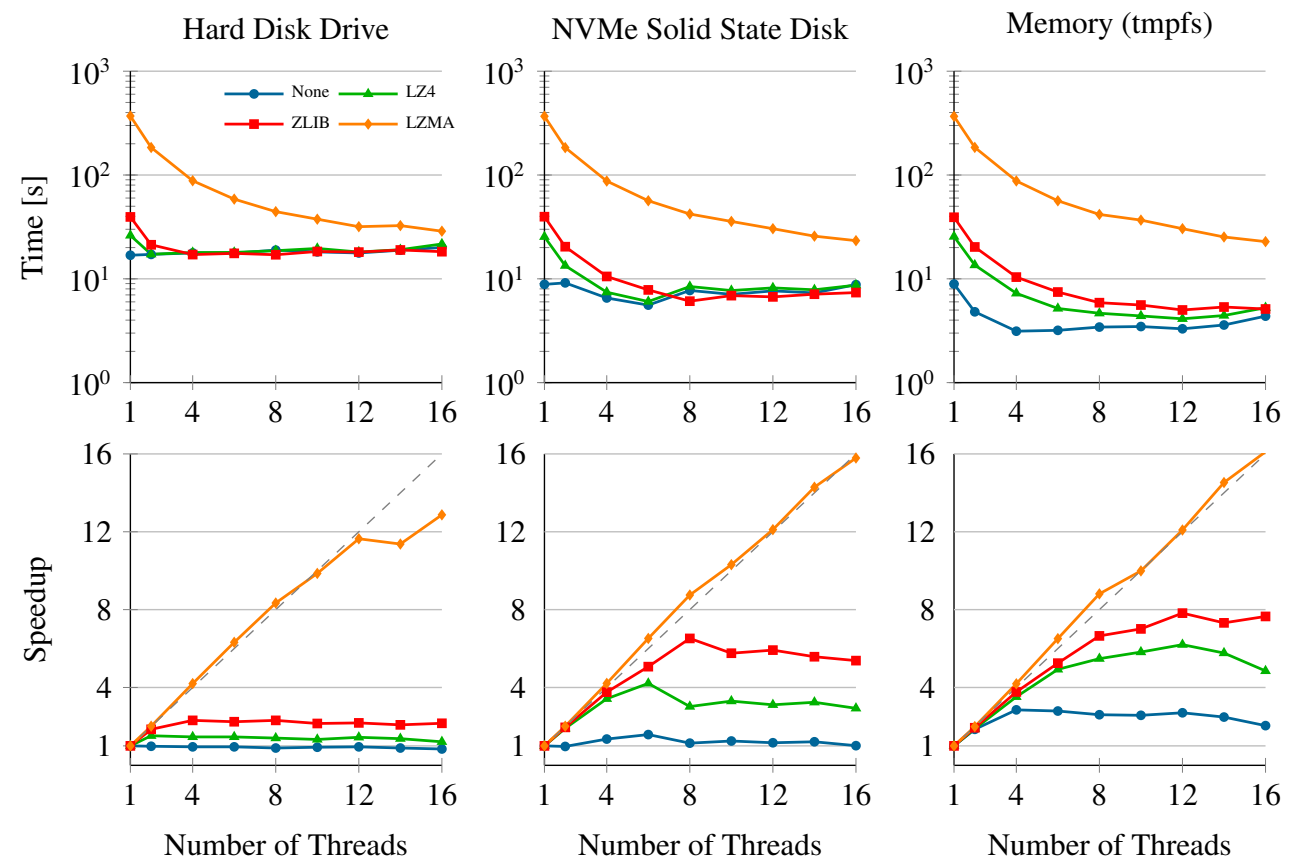

Figure 4: Run time and speedup for creating and saving $\approx 1 \mathrm{~GB}$ of randomly generated floating point numbers in various configurations.

In the next benchmark we increase the role of serialisation in the $\mathrm{I} / \mathrm{O}$ subsystem by generating a more complex dataset where each column consists of an std: : vector $<$ Event $>$, where Event is a class type that itself consists of 3 vectors ( 3 double precision numbers) and 6 other numbers (for a total of $3 \times 3+3=12$ doubles +3 integers). In each run, the number of columns and the number of elements in each vector is varied. In Figure 5, we compare different compression algorithms and output media like in the previous benchmark, but this time using a dataset containing 10 columns where each entry is a vector with 10 randomly generated Events as elements. 

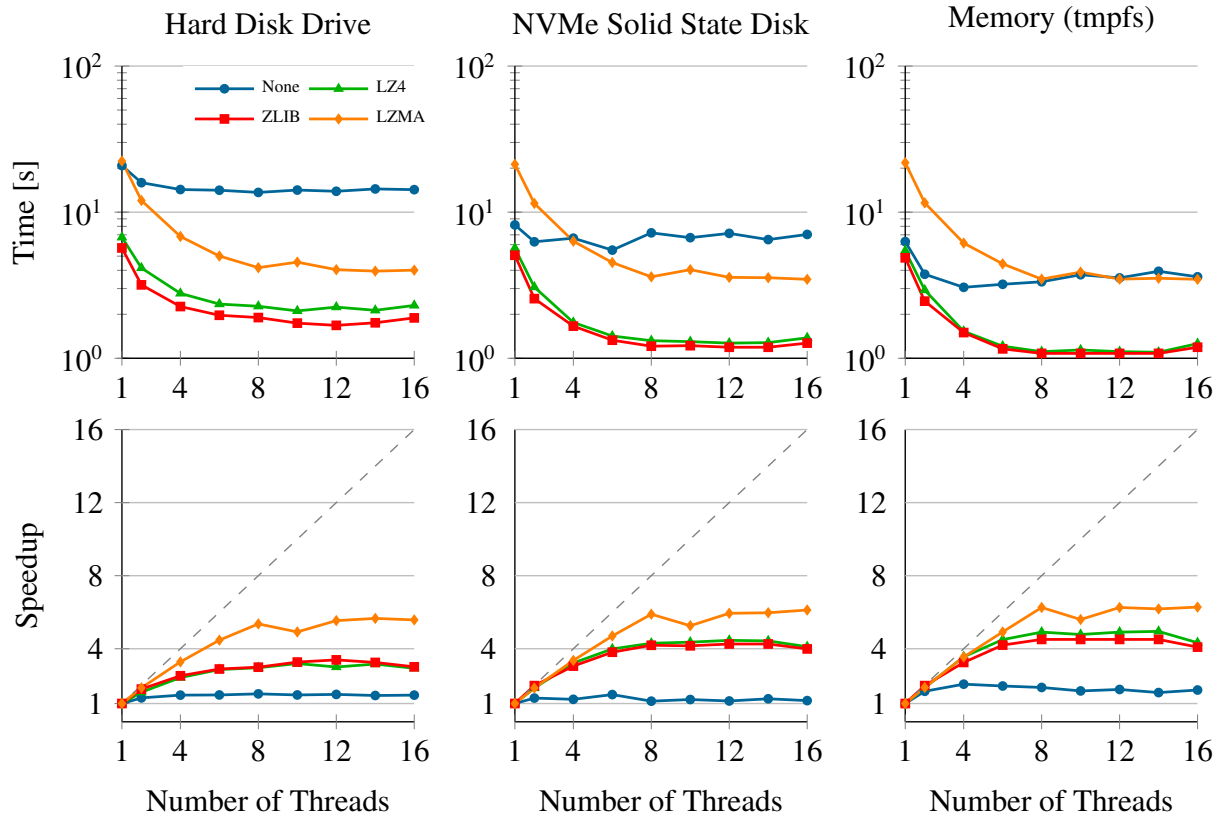

Figure 5: Run time and speedup for creating and saving $\approx 1 \mathrm{~GB}$ of randomly generated std: : vector $<$ Event $>$ in various configurations. Each dataset has 10 columns with vectors of 10 elements as entries.

1 column, 100 elements
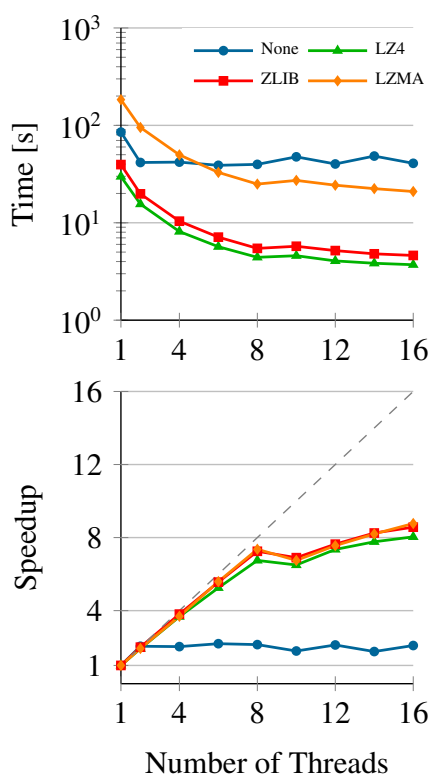

10 columns, 10 elements
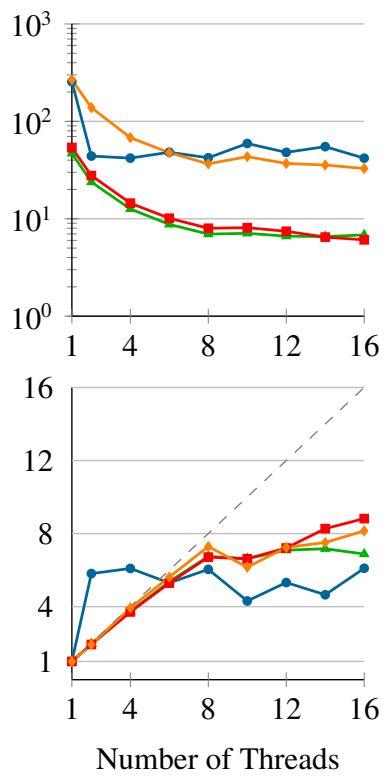

25 columns, 4 elements
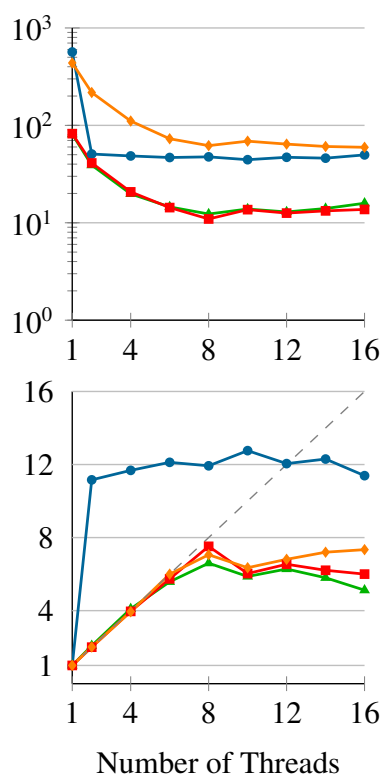

Figure 6: Run time and speedup for creating and saving $\approx 10 \mathrm{~GB}$ of randomly generated std: : vector $<$ Event $>$. Datasets have different numbers of columns and entries, but the total amount of data is kept constant. Only the NVMe solid state disk has been used for data output. 
In the benchmarks of Figure 5, implicit multi-threading (IMT) has been disabled in ROOT. Parallelism is achieved via multiple threads or tasks writing to TBufferMergerFiles, without noticeable differences when using standard threads or TBB tasks for the workers. The time necessary to flush disk buffers after each run is also negligible compared with the run time of each benchmark.

Figure 6 shows that the difference in run time is no longer limited by the bandwidth of the output media, even if it is visibly affected by it. A noticeable difference from the previous benchmark is that LZMA compressed data can be written out to disk faster than uncompressed data due to the higher compression ratio. In all benchmarks, no big difference in performance is observed between ZLIB and LZ4 compression algorithms. In Figure 6, output is written only into the Samsung Evo 960 NVMe SSD, and the flushing strategy is different than before. Per worker, each $1 \%$ of data generated causes a flush event (compression of memory buffers), and each $10 \%$ causes data to be written out from memory buffers to disk. Implicit multi-threading remains disabled, and the size of the generated dataset is increased ten-fold, to about 10GB. The number of data columns is varied along with the number of elements in each vector in such a way that the total amount of generated data remains constant. With the new approach for flushing and saving the data, scaling is improved, but only up to the number of physical cores available on the machine.

\section{Conclusion}

We presented the TBufferMerger class introduced in ROOT 6.10 and used it to characterize the performance of ROOT's I/O subsystem in various scenarios. We have shown that strong scaling can be nearly ideal when the workload is CPU-bound, but that the cost of serialization can become a bottleneck when writing out more complex datasets. In particular, writing out uncompressed datasets has poorer scaling in part due to the higher volume of data, but also due to the heavier relative weight of $\mathrm{I} / \mathrm{O}$ operations given the lack of compression. In realistic workloads, such as simulation and reconstruction, ROOT's I/O subsystem is unlikely to become a performance bottleneck. In CMS reconstruction jobs, for instance, the event processing rate drops only by $1.3 \%$ when writing out results to disk compared with not writing anything (4.59 events/s vs 4.65 events/s) [4]. Performance issues identified by these benchmarks (lock contention during accesses to ROOT's type system) have been fixed and will be available in the upcoming release of ROOT, 6.16/00.

\section{References}

[1] R. Brun, F. Rademakers, ROOT - An Object Oriented Data Analysis Framework (see also ROOT [software], Release v6.16/00), in New computing techniques in physics research V. Proceedings, 5th International Workshop, AIHENP '96, Lausanne, Switzerland (1996)

[2] CERN Computing Page, https://home.cern/about/computing

[3] G. Amadio, B.P. Bockelman, P. Canal, D. Piparo, E. Tejedor, Z. Zhang, Journal of Physics: Conference Series 1085, 032014 (2018)

[4] D. Riley, CMS and ROOT I/O, in ROOT I/O Workshop (2018), https: //indico.cern. ch/event/715802/contributions/2942558

[5] CMSSW Framework, https://github.com/cms-sw/cmssw

[6] J. Blomer, Journal of Physics: Conference Series 1085, 032020 (2018) 